九州大学学術情報リポジトリ

Kyushu University Institutional Repository

\title{
The Expression of Genes Related to Proline Metabolism in Rice (Oryza sativa L.) Under Drought Stress and Re-Watering Conditions
}

\section{DIEN, Doan Cong}

Laboratory of Plant Nutrition, Graduate School of Bioresource and Bioenvironmental Sciences, School of Agriculture, Kyushu University

MOCHIZUKI, Toshihiro

Laboratory of Agroecology, Department of Bioresource, Sciences, Faculty of Agriculture, Kyushu University

ABIK0, Tomomi

Laboratory of Agroecology, Department of Bioresource Sciences, Faculty of Agriculture, Kyushu University

YAMAKAWA, Takeo

Department of Agricultural Science and Technology, Faculty of Agriculture, Setsunan University

https://doi.org/10.5109/4103697

出版情報: 九州大学大学院農学研究院紀要. 65 (2)，pp.237-242，2020-09. Faculty of Agriculture， Kyushu University

バージョン :

権利関係 : 


\title{
The Expression of Genes Related to Proline Metabolism in Rice (Oryza sativa L.) Under Drought Stress and Re-Watering Conditions
}

\author{
Doan Cong DIEN ${ }^{1 *}$, Toshihiro MOCHIZUKI ${ }^{2}$, Tomomi ABIKO ${ }^{2}$ and Takeo YAMAKAWA
}

Laboratory of Plant Nutrition, Division of Molecular Biosciences, Department of Biosciences \& Biotechnology, Faculty of Agriculture, Kyushu University, 744 Motooka Nishi-ku, Fukuoka 819-0395, Japan (Received May 8, 2020 and accepted May 27, 2020)

\begin{abstract}
This study was conducted to investigate the expression of genes related to proline metabolism under drought stress and re-watering conditions in two rice varieties DA8 (drought tolerant) and Malagkit Pirurutong (drought sensitive). The results confirmed that proline was higher accumulated under drought stress than control in both varieties. Under the same drought severity, tolerant variety (DA8) accumulated higher proline than sensitive variety (Malagkit Pirurutong). The expression of P5CS1 and P5CS2, responsible for the synthesis of P5CS enzyme, which catalyzes the first step of the proline synthesis, were induced by drought stress. DA8 showed higher expressions of P5CS2, P5CR (which codes enzyme converting P5C into proline) and OSOAT (involve in proline synthesis through ornithine) than those in Malagkit Pirurutong under severe drought condition. The results suggested that high expression of P5CS1, P5CS2, P5CR and OsOAT genes, particularly under severe drought condition, were in line with the high accumulation of proline. At 4 days after re-watering, proline was quickly degraded due to the decreased expression of synthesis genes and the increased expression of $\mathrm{P} 5 \mathrm{CDH}$, a gene related to proline degradation after re-watering.
\end{abstract}

Key words: drought tolerance, gene expression, proline, rice

\section{INTRODUCTION}

Throughout their life cycle, plants are subjected to various types of environmental stresses, which include salinity, water deficit, temperature extremes, toxic metal ion concentration and UV radiations. These abiotic stresses limit the growth and productivity of plants to varying degrees, depending upon severity of stress. In response to different stresses, plant accumulate large quantities of different types of compatible solutes.

Results of our previous experiments (Dien et al., 2019) showed that proline was highly accumulated under drought stress condition but also quickly degraded after re-watering. These results suggested that proline positively contributed to drought tolerance in rice varieties. Although proline was accumulated in both leaves and sheaths, it was higher accumulated in leaves than in sheaths. This suggested that studies in metabolism of proline should be conducted in leaves.

In plants, proline can be synthesized through the glutamate and ornithine pathways. Glutamate is reduced to glutamate semialdehyde (GSA) through the action of the pyrroline-5-carboxylate synthetase (P5CS) enzyme, and spontaneously converted to pyrroline-5carboxylate (P5C). P5C is reduced to proline by the pyrroline-5-carboxylate reductase (P5CR) enzyme, in the cytoplasm or chloroplast (Rejeb et al., 2014).

\footnotetext{
1 Laboratory of Plant Nutrition, Graduate School of Bioresource and Bioenvironmental Sciences, School of Agriculture, Kyushu University

2 Laboratory of Agroecology, Department of Bioresource Sciences, Faculty of Agriculture, Kyushu University

Present address: Department of Agricultural Science and Technology, Faculty of Agriculture, Setsunan University, 45-1 Nagaotoge-cho, Hirakata City, Osaka, 573-0101, Japan

* Corresponding author: (E-mail: diendoancong@gmail.com)
}

Alternatively, proline may also be synthesized from ornithine through ornithine-delta-aminotransferase (OsOAT) enzyme. OsOAT is a nuclear encoded, pyridoxal-5'-phosphate (PLP)-dependent enzyme found in animals, insects, microorganisms and plants. OsOAT catalyses the transfer of the amino group of L-Orn to 2 oxoglutarate, producing L-glutamate gamma-semialdehyde (GSA) and L-Glu. Then, GSA spontaneously cyclizes to form $\mathrm{P} 5 \mathrm{C}$, a common intermediate in proline metabolism (Stranska et al., 2008). The catabolism occurs in the mytochondria, where the enzyme proline dehydrogenase $(\mathrm{PDH})$ converts proline to $\mathrm{P} 5 \mathrm{C}$ which, through the $\mathrm{P} 5 \mathrm{C}$ dehydrogenase enzyme (P5CDH), produces glutamate (Rejeb et al., 2014). Usually, P5CS is coded by two genes P5CS1 and P5CS2, while P5CR is coded by only one gene P5CR (Armengaud et al., 2004). Likewise, $\mathrm{PDH}$ is transcribed by two genes ( $P D H 1$ and $P D H 2)$, while only one gene $(P 5 C D H)$ codes for $\mathrm{P} 5 \mathrm{CDH}$ (Ribarits et al., 2007).

In order to explore the metabolism of proline synthesis and catabolism, this experiment was conducted to analyzed the expression of genes related to proline metabolism under drought stress and re-watering condition. The difference in gene expression was also compared between drought tolerant rice variety and drought sensitive one.

\section{MATERIALS AND METHODS}

\section{Experimental design}

Two rice varieties (Oryza sativa L.) were used for this experiment including DA8 and Malagkit Pirurutong. Based on results of previous experiment (Dien et al., 2017), DA8 is considered as drought tolerant variety while Malagkit Pirurutong is a drought sensitive one.

Seeds of both varieties were disinfected with $70 \%$ 
(v/v) alcohol for $2 \mathrm{~min}$ and 3\% (v/v) of sodium hypochlorite solution for $25 \mathrm{~min}$, followed by three washings with distilled water. After that, seeds were incubated at $30^{\circ} \mathrm{C}$ for 3 days for germination. Uniformed germinated seeds were sown in plastic cups (height $\times$ width $=105 \times$ $88 \mathrm{~mm}$ ) containing water saturated soil (sandy loam soil, water holding capacity was $47.73 \%$ ). Three germinated seeds of each variety were simultaneously sown in the same cup in order to remain the same soil moisture content between two varieties. After sowing seeds, the cups were placed in a controlled growth chamber (MLR-352PJ, Panasonic, Japan) with the photoperiod of 16 hour light $\left(28^{\circ} \mathrm{C}\right)$ and 8 hour night $\left(25^{\circ} \mathrm{C}\right)$.

At 1week after sowing, seedlings were thin to remain only 2 plants/variety/experimental cup. Drought treatment was started at 10 days after sowing by withdrawing water from drought treatment cups. Plants in control treatment were remained in well-watered condition. Soil moisture content was measured by soil moisture sensor (EC5, Decagon, USA). Drought treatment was prolonged until soil water content declined to $10 \%$ $(\mathrm{v} / \mathrm{v})$, then water is re-supplied for recovery of plants.

The first sampling was carried out on drought-treatment day. Further sampling times were performed when soil water content decreased to 30\%, 20\%, 10\% (v/v), respectively. Sampling in recovery stage was conducted at 4 days after re-watering. Fig. 1 showed the timeline in this experiment.

Because proline was mainly accumulated in leaves in comparison with other parts, sampling and analyses in this experiment were performed in leaves only. For each sampling time, there random experimental cup (in three replication) were selected. All leaves of each sampling plants were quickly detached from plant and transferred to a plastic-zip bag. Samples after taken were immediately stored in $-80^{\circ} \mathrm{C}$ for further analysis.

\section{Proline analysis}

Sample were crushed by Micro Smash ${ }^{\mathrm{TM}}$ MS-100 (TOMY, Japan) before used for further analyses. A half amount of crushed sample was used for proline analysis, other half was used for gene expression analysis. Proline content was analysed follow the method of Bates et al.
(1973).

\section{Total RNA extraction and quantitative real-time PCR}

Fresh samples used for gene expression analysis were the same samples used for proline analysis. Total RNA from leaves of two rice varieties were extracted using RNeasy Plant mini kit (QIAGEN, Hildern, Germany) according to the manufacturer's instructions. The cDNA was synthesized from $500 \mathrm{ng}$ of total RNA using Takara PrimeScript ${ }^{\mathrm{TM}}$ RT-PCR Kit (Takara Bio Inc., Japan) according to the manufacture's protocol. In order to take exactly amount of template RNA, we measured concentration of extracted RNA by nanodrop lite spectrophotometer (Thermo Fisher Scientific Inc., USA) and then diluted RNA with RNase free water.

Real-time PCR was conducted using the SYBR Premix Ex Taq ${ }^{\mathrm{TM}}$ GC (Takara Bio Inc., Japan) in a total volume of $20 \mu \mathrm{L}$ by Rotor-Gene Q (QIAGEN, Hildern, Germany). The PCR conditions were one cycle of $95^{\circ} \mathrm{C}$ for $30 \mathrm{~s}$ followed by 40 cycles of $95^{\circ} \mathrm{C}$ for $5 \mathrm{~s}$ and $60^{\circ} \mathrm{C}$ for $30 \mathrm{~s}$ according to the manufacture's instruction. The OsUBQ10 gene was used as internal control (Moraes et al., 2015). The primers for the genes used are listed in Table 1. The relative quantification of differential expression was made using the CT method (comparative threshold cycle method), as described by Livak and Schmittgen (2001) with three replicates.

\section{Statistical analysis}

The experiment was conducted in a randomized complete block design with 3 replications. Analysis of variance was used to test for differences, and Turkeys's HSD test was used to calculate the significant difference at the 5\% probability level using STAR 2.0.1 software.

\section{RESULTS}

\section{Soil water content}

At 10 days after seed sowing, drought treatment was commenced by withdrawing water from drought-treatment cup. Fig. 2 demonstrate the changing of soil water content (SWC) during drought stress period. SWC in

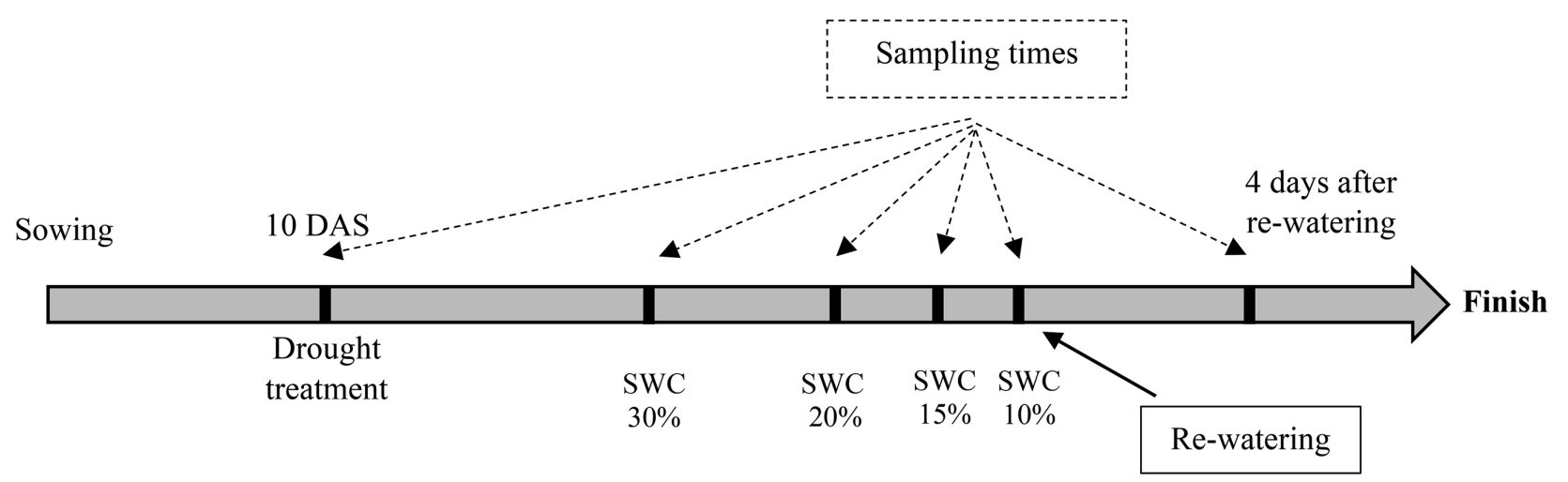

Fig. 1. Timeline in this experiment. 
Table 1. List of primers used in RT-qPCR

\begin{tabular}{lll}
\hline Gene & Primer & Sequence 5'-3' \\
\hline P5CS1 & Forward & AGAGATTGTTCAAGGCATCTACAG \\
& Reverse & AACCAAAGGCTTCTCATATCCAG \\
P5CS2 & Forward & GGTTGATTATCCAGCAGCGT \\
& Reverse & CCCGCCATAGATAACTACCC \\
P5CR & Forward & GTTGGTCTGGTCATCGAAGATT \\
& Reverse & CATTCTCAGTAGCCATCTCCC \\
P5CDH & Forward & AGCAGAGGATGTGGACTTCA \\
& Reverse & GCCTTTCAAATCAGCAGCC \\
PDH & Forward & GGGTTCCAGGTGAGCAAG \\
& Reverse & AAGGAGGAAGACGAGAGCA \\
OsOAT & Forward & TGCTGTGTATCAAACCAGGA \\
& Reverse & CTTTCAACGAGACCTTCATCTG \\
UBQ10 & Forward & TGGTCAGTAATCAGCCAGTTTGG \\
& Reverse & GCACCACAAATACTTGACGAACAG \\
\hline
\end{tabular}

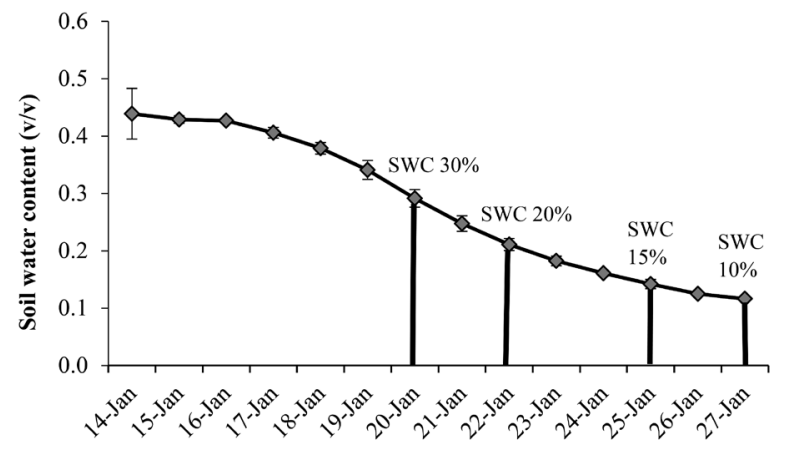

Fig. 2. Soil water content during drought stress period. SWC: Soil Water Content; Average \pm standard deviation $(n=24)$.

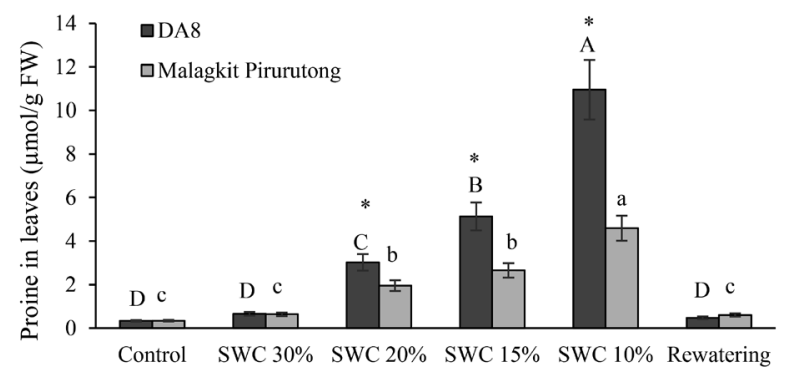

Fig. 3. Proline content in leaves of varieties under different water conditions.

SWC: Soil Water Content (volumetric \%). * Significant difference between DA8 and Malagkit Pirurutong at $p<0.05$; Capital letters and lowercase letters indicate significant difference between different water conditions in DA8 and Malagkit Pirurutong, respectively. Average \pm standard error $(n=3)$. drought-treatment cup gradually decreased through the increasing of drought severity. At 6 days after drought treatment, SWC decreased to around 30\% (v/v). SWC declined to only $10 \%(\mathrm{v} / \mathrm{v})$ at 2 weeks after drought treatment. In this experiment, in addition to control and re-watering, samples were taken when SWC decreased to $30 \%, 20 \%, 15 \%$ and $10 \%(\mathrm{v} / \mathrm{v})$.

\section{Proline content}

Proline content in leaves of two rice varieties was presented in Fig. 3. Under control condition and 30\% of soil water content, proline was accumulated at low concentration and there was no significant difference between two varieties. Until SWC 30\%, there was no significant increase of proline content in leaves of DA8 and Malagkit Pirurutong. Corresponding with the increasing of drought severity, proline content increased in both varieties. At SWC 10\%, proline reached the highest concentration in leaves of DA8 and Malagkit Pirurutong at 10.95 and $4.59 \mu \mathrm{g} \mathrm{g}^{-1} \mathrm{FW}$, respectively. From SWC 20\% to SWC $10 \%$, proline was higher accumulated in leaves of DA8 than that in Malagkit Pirurutong. Proline content in leaves of both varieties were significantly decreased in re-watering condition and reached to the same level of control condition. There was no difference in proline content between two rice varieties in re-watering condition.

\section{Expression of proline-related genes}

Fig. 4 present the expression of genes related to proline metabolism under different water conditions. As shown in Fig. 4, the expression of P5CS1 and P5CS2 genes, responsible for the synthesis of the pyrroline-5carboxylate synthetase (P5CS) enzyme, which catalyzes the first step of the proline biosynthesis, was induced by drought stress. The highest expression of P5CS1 was attained at SWC 10\% for DA8 and SWC 15\% for Malagkit Pirurutong, respectively. At SWC 30\% and SWC 15\%, relative expression of P5CS1 in Malagkit Piruruton were higher than those in DA8. However, under severe drought stress (SWC 10\%), there was no significant difference in gene expression of P5CS1 between two varieties. After re-watering, relative expression of P5CS1 decreased to only 0.65 and 0.67 fold in DA8 and Malagkit Pirurutong, respectively.

The expression of P5CS2 under drought condition was quickly increased compared to control condition. Both varieties DA8 and Malagkit Pirurutong showed the highest relative expression of P5CS2 at SWC 10\%. In comparison between two varieties, DA8 showed the higher expression of P5CS2 than Malagkit Pirurutong at SWC 15\% and SWC 10\%. During the drought stress from SWC 15\% to SWC 10\%, DA8 (but not Malagkit Pirurutong) showed the increasing of gene expression of P5CS2 (from 5.71 to 13.83 fold). Similar to P5CS1, the expression of P5CS2 decreased after re-watering.

At the early stage of drought stress (SWC 30\%), the relative expression of $P 5 C R$ in Malagkit Pirurutong was higher than in DA8. However, the opposite trend was observed in severe drought stress. At SWC 15\% and 

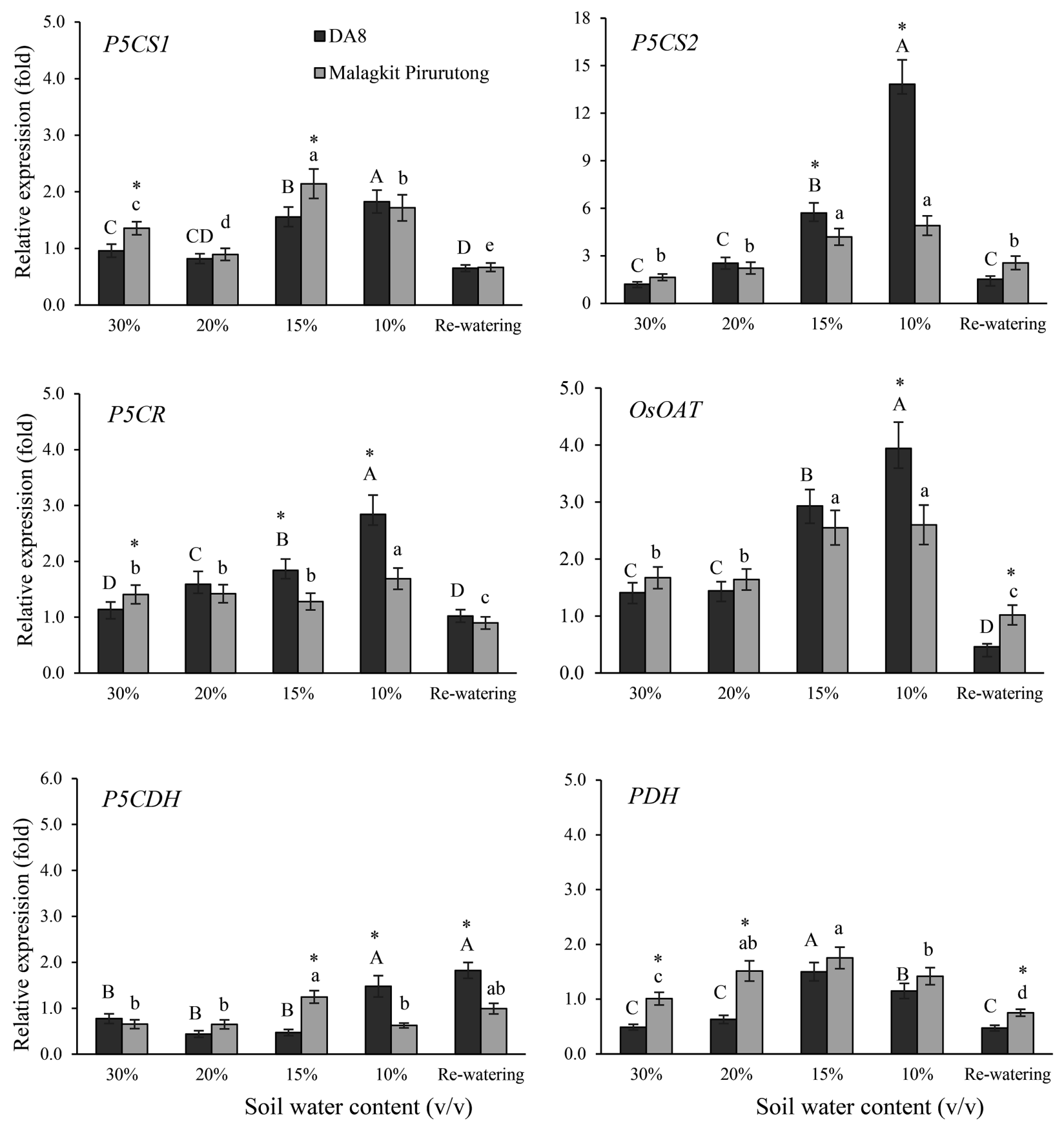

Fig. 4. Expression of genes related to proline metabolism under drought stress and re-watering.

* Significant difference between DA8 and Malagkit Pirurutong in the same water condition at $p<0.05$; Capital letters and lowercase letters indicate significant differences between different water conditions in DA8 and Malagkit Pirurutong, respectively. Average \pm standard error $(n=3)$. 
$10 \%$, the relative expression of P5CR in DA8 (1.84 and 2.84 fold, respectively) were significantly higher than those in Malagkit Pirurutong (1.28 and 1.69 fold, respectively). After re-watering from drought stress, the expression of $P 5 C R$ decreased in both varieties.

Relative expression of gene OsOAT under drought stress was higher than control in both varieties. The expression of OSOAT quickly increased during drought stress from SWC 20\% to SWC 10\%. Malagkit Pirurutong showed the highest relative expression of OsOAT at SWC 15\% and not increased until the end of drought treatment. In cased of DA8, OsOAT reached the highest expression level at SWC 10\% (3.94 fold, and significantly higher than that in Malagkit Pirurutong). The relative expression of OSOAT decreased after re-watering. Interestingly, the relative expression of Malagkit Pirurutong was higher than that in DA8 at recovery stage.

At SWC 15\%, the relative expression of $P 5 C D H$ in Malagkit Pirurutong reached the highest level (1.25 fold), significantly higher than DA8. The relative expression of $P 5 C D H$ in DA8 remained low levels from SWC $30 \%$ to SWC $15 \%$, before increased to 1.48 fold at SWC 10\% and highest level at re-watering (1.82 fold). DA8 showed the higher expression level than Malagkit Pirurutong at SWC 10\% and re-watering.

At SWC 30\% and SWC 20\%, relative expression level of $P D H$ in DA8 was lower than control. However, this expression level was increased and reached the highest value at SWC 15\% before gradually decreased at SWC $10 \%$ and re-watering. In Malagkit Pirurutong, the relative expression of $P D H$ was significantly higher than those in DA8 at SWC 30\% and SWC 20\%. Malagkit Pirurutong also demonstrated the highest expression level of $P D H$ at SWC 15\%, however there was no significantly difference between two rice varieties at this time. At re-watering condition, the expression of $P D H$ in Malagkit Pirurutong was higher than that in DA8.

\section{DISCUSSION}

In this experiment, rice plants grown under drought condition showed higher proline concentration than control and this concentration increased through the increasing of drought severity (Fig. 3). The result consistent with our finding in previous study (Dien et al., 2019). In order to understand the proline metabolism, the expression of genes involved in the metabolism of this amino acid were evaluated. As shown in Fig. 4, the expression of P5CS1 and P5CS2 genes, responsible for the synthesis of P5CS enzyme, which catalyses the first step of the proline biosynthesis, was induced by drought stress, particularly at SWC 15\% and SWC 10\%. This results agreed with previous studies (Suravoot et al., 2012; Tatiana et al., 2018) which indicated that P5CS1 and P5CS2 increased the gene expression under drought and salt stress conditions. In this experiment, P5CS1 showed the higher expression in Malagkit Pirurutong than in DA8 (at SWC 30\% and SWC 15\%). In contrast, DA8 showed the higher expression of P5CS2 compared to Malagkit Pirurutong, at SWC 15\% and SWC 10\%. In Fig.3, DA8 accumulated higher proline content than Malagkit Pirurutong, particularly in SWC 10\%. This suggested that in DA8, P5CS2 is the main gene which responsible for the synthesis of P5CS enzyme.

The highest expression of $P 5 C R$, which codes for the enzyme converting $\mathrm{P} 5 \mathrm{C}$ into proline, occurred at the highest stress (SWC 10\%) in both DA8 and Malagkit Pirurutong. In previous study, Hsu et al. (2003) found that $P 5 C R$ activities decreased in plants subjected to water deficit. In contrast, Parida et al. (2008) suggested that the activity of P5CR in drought tolerant genotypes were superior to those in drought susceptible. In this experiment, Malagkit Pirurutong (a drought sensitive rice variety) showed a higher expression of $P 5 C R$ than DA8, a drought tolerant one at early stage of drought stress (SWC 30\%). However, the opposite trend was observed under severe drought stress (SWC 15\% and SWC 10\%), DA8 expressed higher expression of P5CR than Malagkit Pirurutong (Fig. 4).

Through the duration of drought stress, it was verified that mRNA levels of OsOAT gene was significantly higher than control. In a previous study, Jun et al. (2012) found that OsOAT gene increased drought and oxidative stress tolerance in rice mainly through enhancing ROS-scavenging capacity and proline pre-accumulation. In this experiment, OsOAT expression level was higher than control throughout the drought duration and reached the highest expression level at SWC 10\%.

Regarding the represented expression of genes related to proline degradation, it can be observed that $P 5 C D H$ gene, which codes for an enzyme that converts PC5 to glutamate, showed maximum expression at SWC $15 \%$ in Malagkit Pirurutong. In DA8, the expression of $P 5 C D H$ reached the highest level at SWC 10\% and remained this level to re-watering stage. Tatiana et al. (2018) suggested that the expression of P5CDH gene reached the highest level at 20 days under salt stress condition. In case of $P D H$, Malagkit Pirurutong showed the higher expression than DA8 through the drought duration and re-watering also. The expression of $P D H$ in both DA8 and Malagkit Pirurutong were reached the highest levels at SWC 15\% (Fig. 4).

Based on the results of gene expression throughout drought stress, it was deduced that the elevated levels of proline under drought stress condition are due to the higher expression of genes involved in the proline biosynthesis, when compared with genes that code for catabolism enzymes. In this experiment, high expression levels of the P5CS2, P5CR and OAT genes, particularly at SWC $10 \%$, are in line with the high concentrations of proline in this period. It proposed that proline biosynthesis by glutamate and ornithine prevails under drought stress condition in rice varieties.

Several studies have shown that P5CS transcription in plants, although varies according to the species, is promoted mainly by adverse environmental conditions like drought, salinity, and high and low temperatures. This our study showed increased expression of both P5CS1 and P5CS2, although the expression of P5CS1 at 
lower levels compared to P5CS2. Similar results were found in rice by Hur et al. (2004). According to these authors, both P5CS1/P5CS2 responded in the plants grown under stress. The authors concluded that the P5CS1 gene is involved in maintenance, providing a basal level of proline to the cell, while P5CS2 is the first to respond to stressed conditions (Tatiana et al., 2018).

During proline biosynthesis, P5C when accumulated in excess, can cause reactive oxygen species (ROS) production and induce apoptosis causing cell death (Szekely et al., 2008). Therefore, plants need to degrade it rapidly, as soon as the tension is relieved. Proline catabolism occurs in the mitochondria through the action of $\mathrm{PDH}$ or proline oxidase (POX) producing $\mathrm{P} 5 \mathrm{C}$, which is converted to glutamate through $\mathrm{P} 5 \mathrm{CDH}$ (Szabados and Savouré, 2010).

At 4 days after re-watering, the expression of P5CS1, P5CS2, P5CR and OsOAT in both rice varieties significantly decreased compared to drought stress condition. Plant accumulated proline under drought stress condition, but once the stress is withdrawn, proline is oxidized to P5C by PDH, the first enzyme in the proline degradation pathway (Kishor et al., 2005). This also explained why the expression of $\mathrm{P} 5 \mathrm{CDH}$ was at high level after re-watering.

In conclusion, this experiment confirmed that proline was highly accumulated under drought stress condition but quickly degraded after re-watering. The analysis of gene expression explored the mechanism of proline metabolism. Under drought stress condition, the expression of P5CS1, P5CS2, OsOAT and P5CR increased the synthesis of proline through the glutamate and ornithine pathways. Among genes involved in proline biosynthesis, the expression of P5CS2 and OsOAT contributed to the higher proline accumulation in DA8 than in Malagkit Pirurutong, particularly at SWC 10\%. After re-watering, the expression of these genes quickly decreased to very low levels, accompanied with the expression of $P 5 C D H$ and $P D H$ genes, reached to the decreasing of proline content in leaves of rice varieties.

\section{AUTHOR CONTRIBUTIONS}

Doan Cong DIEN designed the study, gathered and analysed statistically the data, and wrote the first draft of the manuscript. Tomomi ABIKO managed the analysis of parameters in the study. Takeo YAMAKAWA designed the study, managed the analysis of parameters in the study. Takeo YAMAKAWA and Toshihiro MOCHIZUKI managed the literature search and edited the manuscript. All authors read and approved the final manuscript.

\section{ACKNOWLEDGMENT}

This research was supported by a Monbukagakusho Scholarship from the Japanese Ministry of Education, Culture, Sports, Science and Technology (MEXT). The authors would like to thank Agricultural Ecology Laboratory, Faculty of Agriculture, Kyushu University, for providing research facilities for this study.

\section{REFERENCES}

Armengaud, P., Thiery, L., Buhot, N., March, G. G. D. and Savouré, A. 2004. Transcriptional regulation of proline biosynthesis in Medicago truncatula reveals developmental and environmental specific features. Physiologia Plantarum, 120: 442-450

Bates, L. S., Waldren, R. P. and Teare, I. D. 1973. Rapid determination of free proline for water-stress studies. Plant and Soil, 39: 205. https://doi.org/10.1007/BF00018060

Dien, D. C., Mochizuki, T. and Yamakawa, T. 2017. Morphology and dry matter accumulation in rice (Oryza sativa L.) seedlings under drought conditions. Journal of the Faculty of Agriculture, Kyushu University, 62(2): 309-322

Dien, D. C., Thu, T. T. P., Moe, K. and Yamakawa, T. 2019. Proline and carbohydrate metabolism in rice varieties (Oryza sativa L.) under various drought and recovery conditions. Plant Physiology Reports, 24: 376-387

Hsu, S., Hsu, Y. and Kao, C. 2003. The effect of polyethylene glycol on proline accumulation in rice leaves. Biologia Plantarum, 46: 73-78. https://doi.org/10.1023/A:1022362117395

Jun, Y., Honghong, H. and Lizhong, X. 2012. An ornithine $\delta$-aminotransferase gene OsOAT confers drought and oxidative stress tolerance in rice. Plant Science, 197: 59-69

Kishor, P. B. K., Sangam, S., Amrutha, R. N., Laxmi, P. S., Naidu, K. R., Rao, K. R. S. S. et al. 2005. Regulation of proline biosynthesis, degradation, uptake and transport in higher plants: Its implications in plant growth and abiotic stress tolerance. Current Science, $\mathbf{8 8}(3)$ : 424-438

Livak, K. J. and Schmittgen T. D., 2001. Analysis of relative gene expression data using real-time quantitative PCR and the $2(-$ Delta Delta C(T)) method. Methods, 25: 402-408

Moraes, G. P., Benitez, L. C., Amaral, M. N., Vighi, I. L., Auler, P. A., Maia, L. C., Bianchi,V. J. and Braga, E. J. B. 2015. Evaluation of reference genes for RT-qPCR studies in the leaves of rice seedlings under salt stress. Genetics and Molecular Research 14(1): 2384-2398

Parida, A. K., Dagaonkar, V. S., Phalak, M. S. and Aurangabadkar, L. P. 2008. Differential responses of enzymes involved in proline biosynthesis and degradation in drought tolerant and sensitive cotton genotypes during drought stress and recovery. Acta Physiologiae Plantarum, 30: 619-627

Rejeb, K. B., Abdelly, C. and Savouré, A. 2014. How reactive oxygen species and proline face stress together. Plant Physiology Biochemistry, 80: 278-284

Ribarits, A., Abdullaev, A., Tashpulatov, A., Richter, A, HeberleBors, E. and Touraev, A. 2007. Two tobacco proline dehydrogenases are differentially regulated and play a role in early plant development. Planta, 225(5): 1313-1324. doi:10.1007/s00425006-0429-3

Stranska, J., Kopecny, D., Tylichova, M., Snegaroff, J. and Sebela, M. 2008. Ornithine $\delta$-aminotransferase: An enzyme implicated in salt tolerance in higher plants. Plant Signaling and Behavior, 3(11): 929-935

Suravoot, Y., Suriyan, C. and Kanyaratt, S. 2012. Proline related genes expression and physiological changes in indica rice response to water-deficit stress. Plant Omics Journal, 5(6): 597-603

Szabados, L. and Savouré, A. 2010. Proline: a multifunctional amino acid. Trends in Plant Science, 15(2): 89-97

Szekely, G., Abraham, E., Cselo, A., Rigo, G., Zsigmond, L., Csiszar, J. et al. 2008 Duplicated P5CS genes of Arabidopsis play distinct roles in stress regulation and developmental control of proline biosynthesis. Plant Journal, 53: 11-28

Tatiana, R., Mara, A. C. M., Marcelo, N. A., Priscila, A. A., Rafael, W., Ariano, M. M. J et al. 2018. Morphophysiological analysis and expression of proline genes in rice (Oryza sativa L. cv. BRS AG) subjected to in vitro salt stress. Australian Journal of Crop Science, 12(02): 243-253 